\title{
Compression-Induced Solidification: A Novel Processing Technique for Precise Thermoplastic Optical Components with Negligible Internal Stresses
}

\author{
Ariane Jungmeier, ${ }^{1}$ Wolfgang Wildner, ${ }^{1}$ Dietmar Drummer, ${ }^{1}$ and Ines Kühnert ${ }^{2}$ \\ ${ }^{1}$ Institute of Polymer Technology, Friedrich-Alexander-University Erlangen-Nürnberg, Am Weichselgarten 9, 91058 Erlangen, Germany \\ ${ }^{2}$ Leibniz Institute of Polymer Research Dresden, Hohe Straße 6, 01069 Dresden, Germany
}

Correspondence should be addressed to Ariane Jungmeier, jungmeier@lkt.uni-erlangen.de

Received 1 March 2012; Accepted 6 May 2012

Academic Editors: D. Kouznetsov and V. Toal

Copyright ( $) 2012$ Ariane Jungmeier et al. This is an open access article distributed under the Creative Commons Attribution License, which permits unrestricted use, distribution, and reproduction in any medium, provided the original work is properly cited.

\begin{abstract}
In the field of optical components, thermoplastics are replacing more and more glass mainly because of their better freedom of design and their cost-effective processing techniques. Nevertheless, especially lenses do not have an ideal design for plastic processing, because of their varying thickness from the centre to the edge. These lead to great differences in shrinkage due to the different coefficients of thermal expansion of melt and solid state and, consequently, directly lead to warpage and residual stresses with state-of-the-art processing techniques. A promising solution is a new, innovative technique-compression-induced solidification (CIS) - where the melt is compressed at constant temperature until it solidifies. This results in isochronic solidification of the whole part even at high temperatures and reduces residual stresses and warpage due to the cooling of a body with homogenous shrinkage. In this paper, CIS integrated in the injection molding process is introduced, and the influence of process parameters on inner properties and dimensional accuracy of CIS polycarbonate parts are illustrated. Trials carried out indicate that an optimum level of compression pressure at the end of glass transition range and a sufficiently long period of holding time (hereinafter the adapting time) for reaching homogeneous temperatures within the melt until pressure is applied will generate parts with low residual stresses and high dimensional accuracy.
\end{abstract}

\section{Introduction}

Optical components for high-precision applications, especially in the case of complex geometries, are mostly made of glass by expensive and time-consuming grinding and polishing techniques. For that purpose, plastics offer the possibility of cheap mass production and easily achievable complex geometries. The state of technology for the production of plastic parts are injection and injection compression molding. After mold filling in the injection molding process, cooling-dependent shrinkage is balanced by the packing pressure that is held until material in the the sprue freezes and the material flow in the cavity is inhibited. From that moment on, the molded part will shrink according to pressure- and to specific volume- and temperature- ( $p v T-$ ) dependent behavior. Regions of accumulated material will freeze later than thinner parts and layers that are adjacent to the cold mold walls. The liquid state, with its thermal expansion coefficient generally at least two times higher than the solid state, will lead to inhomogeneous shrinkage over the whole part, as well as shrink marks and residual stresses.

Refractive lenses are parts with great variations in thickness and are thus only suitable for production by injection molding to a limited extent. To match tolerances, the molds are fitted step by step until the shrink marks are minimized and the parts are within the geometric tolerances. Another processing technology used for the production of optical parts is injection compression molding. As opposed to standard injection molding, the packing pressure is not applied by the screw but by movable parts of the mold. As an effect, the pressure is not varied over the flow path but is homogenously applied over a longer period on the whole 

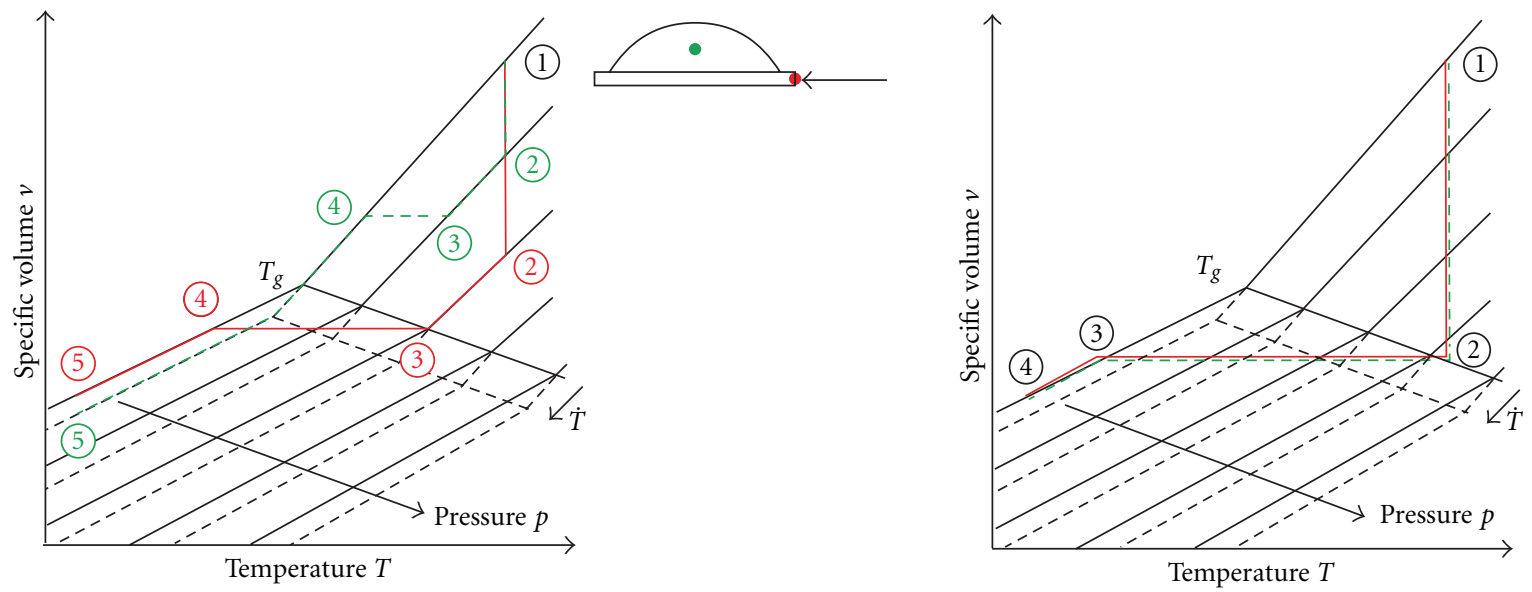

--- Central
- Near the gate

- - - Central

Near the gate

FIGURE 1: Schematic presentation of the course of the pressure- and temperature-related specific volume ( $p v T$ behavior) of a lens made by injection molding (left) and of a lens made by isochoric compression induced solidification (right).

part and can therefore be more effective. Thus shrinkage and residual stresses can be reduced.

A possibility to avoid these effects caused by the simultaneous existence of solid and liquid layers in one part is to separate solidification from cooling. This can be achieved by applying high pressure upon a polymer melt with a homogenous temperature. Due to the $p v T$ behavior, glass transition is dependent on temperature and pressure. Thus the melt solidifies as pressure increases at higher temperatures. Based on this relation, the Institute of Polymer Technology developed a new processing technology for amorphous thermoplastic materials, the compressioninduced solidification (CIS) technique. After homogenous solidification of the melt by pressure, the cooling of the part takes place only in the solid state. In the following, the theoretical background of CIS will be described first. After that, the focus will be on the important subject of implementing a necessary new mold technology in the experimental part, followed by the illustration of the influence of compression pressure and temperature adapting time on the properties of thick-walled parts produced via CIS. In addition, possibilities of the reduction of the long cycle time by optimization of the temperature balance or the process strategy are discussed.

\section{State of the Technology}

2.1. Material Behavior of Amorphous Thermoplastics Relevant to Processing. Plastics' behavior during processing is significantly determined by temperature and pressure. These two parameters are of major importance to density and its inverse value, that is, specific volume. The term $p v T$ behavior of plastics is used here. A $p v T$ measuring device is usually employed to determine this type of behavior. With this technique, for instance, starting from a defined processing temperature, isobaric cooling is conducted, and the values of the specific volume $\mathrm{v}$ sequentially determined against a temperature $T$ at different pressures $p$. Amorphous thermoplastics, which are mainly used for plastics optics, have glass transition temperatures $T_{g}$, or ranges, that extensively restrict the mobility of macromolecules, which are mobile in the molten state, so that the material freezes. Between glass transition and the molten state, the material exhibits entropy-elastic behavior. Subject to the respective material, the process of glass transition may span up to approximately $20^{\circ} \mathrm{C}$. Viscosity rises by several orders of magnitude here [1]. Glass transition itself is determined by the parameters of pressure and cooling speed. As pressure rises, glass transition temperature increases. And higher cooling rates lead to higher glass transition temperatures as well.

Additionally, thermoplastics behavior of thermal expansion changes decisively at glass transition range. The thermal expansion coefficient $\alpha$ in a molten state is generally at least twice as high as that in the solid body. As a result, shrinkage (change in volume) during cooling is significantly higher above glass transition than below the range of glass transition.

2.2. Effects of Pressure and Temperature on the Component Volume Obtained from Injection Molding. In injection molding, only the packing pressure from the plastification unit counteracts the material-inherent shrinkage that takes place during cooling. Here, the molten material remains submitted to pressure until the sprue is frozen. Changes in wall thicknesses, and the temperature gradient over the flow cross-section, lead to inhomogeneous pressure distribution along the flow path, which then affects the properties of the molded part $[2,3]$. A $p v T$ diagram can be seen in Figure 1 (left) showing an example of an ideal case of the course of the specific volume during a conventional injection molding process for a component with great differences 
TABle 1: Properties of the examined PC Makrolon LQ 2647.

\begin{tabular}{lc}
\hline Application & Optical components, lenses \\
\hline Glass transition temperature $\left[{ }^{\circ} \mathrm{C}\right]^{(1)}$ & 143 \\
Density $\left[\mathrm{g} / \mathrm{cm}^{3}\right]^{(2)}$ & 1.2017 \\
MVR $\left(300^{\circ} \mathrm{C}, 1.2 \mathrm{~kg}\right)[\mathrm{g} / \mathrm{min}]^{(3)}$ & 12.5 \\
${ }^{(1)}$ In-house measurement, DSC, 2. heating, $20 \mathrm{~K} / \mathrm{min}$, granulate. \\
${ }^{(2)}$ In-house measurement; gas pycnometer, $23^{\circ} \mathrm{C}$, granulate. \\
${ }^{(3)}$ According to data sheet of supplier $[7]$.
\end{tabular}

in wall thicknesses. Following injection molding (1-2), the unit switches to packing, thus feeding melt until the sprue freezes (3). The process of cooling is almost isobaric, with pressures lower at the thick-walled center of the molded part than at the sprue, due to the pressure decrease over the flow path. As soon as the sprue is frozen (3), so-called isochoric shrinkage starts, which means component volume corresponds to cavity volume and pressure decreases as a result of thermal shrinkage. As atmospheric pressure is reached (4), the component can shrink freely [4]. Because the heat conductivity of plastics is low, the center of the thick-walled component cools down much more slowly. This leads to grave differences in temperatures and specific volumes (4) at the same point in time; while pressures at the center are lower too, the shrinkage potential is higher (green graph). These gradients can generate shrink marks and specific volumes being lower in the thicker areas (5) [5].

\subsection{Effects of the Control of Pressure and Temperature on} the Component Volume Obtained from CIS. Figure 1 (right) shows the course of the temperature- and pressure-related specific volume of a lens made by CIS. Starting from a homogenous melt temperature (1), the pressure is stepped up until glass transition is reached or exceeded (2), respectively, thus making the melt solidify simultaneously in each spot within the component. Melt temperature has to be homogeneous, first, to provide for pressure impact on and distribution in the melt to be homogeneous too and, second, to make sure glass transition is paced at the same time, because the pressure required is significantly determined by temperature. The holding time necessary for cooling down the injected melt in the cavity to compression temperature by heat conduction is called temperature adapting time. Then, after compression, the solid component is cooled down, which is done either at constant volume until environmental pressure is reached (isochoric: $2-3$ ) or at pressure remaining constant at first and then reduced isothermally (isobar). Having reached environmental pressure, the component is finally cooled down to environmental temperature (4). A component solidified in this way, with its thermal contraction of solid-state bodies, features shrinkage that is lower and more homogeneous over the entire cross-section than do parts made by standard injection molding.

\section{Experimental}

The investigations described in the following were aimed at describing the effects exerted during cooling by the process parameters of compression pressure and temperature adapting time on the resulting residual stresses and the dimensions of the component. To determine the limits of densification in the glass transition range and define the densification parameters, the material is submitted to preliminary characterization. Samples are then produced within and outside these limits and their resulting properties evaluated. Component characterization is done following their photoelastic behaviors and geometries.

3.1. Material. The process of CIS requires a thermoplastic material, which provides for the displacement of the glass transition to exceed the compression heating. Polycarbonate (PC) usually meets this requirement. For example, the displacement of the glass transition temperature of the standard PC-type Makrolon 2805 is $0.028 \mathrm{~K} / \mathrm{bar}$ and the compression heating is $0.006 \mathrm{~K} / \mathrm{bar}[6]$. Furthermore, qualification for optical applications is necessary. In the work at hand, the PC-type Makrolon LQ 2647, supplied by Bayer Material Science AG, is used for the investigations. An overview of the material's general properties is illustrated in Table 1 .

3.2. Material Characterization. To determine the compression and temperature-related glass transition range of the used material, compression measurements are conducted in a compression test mold. The process corresponds to the investigations described by Rudolph et al. [1, 4, 8]. Compression measurements up to 3500 bar of cavity pressures are conducted at initial temperatures of 150, 170, 190, 210, and $230^{\circ} \mathrm{C}$ and the generated v-p courses evaluated. As was described by Breuer and Rehage [9], these courses can serve as a basis for the calculation of compression moduli, which will then indicate the beginning and the end of the vitreous solidification caused by compression. The limits to compression solidification thus determined will be employed in the following trials.

\subsection{Sample Production}

3.3.1. New Mold Technology. Based on the previous findings concerning the densification process carried out in a compression test mold, an experimental injection mold was designed for densification, which can be integrated into a conventional injection molding machine (Figure 2). In the work at hand, an Engel Victory 200/60 Electric, by Engel Austria $\mathrm{GmbH}$, Austria, with a clamping force of $600 \mathrm{kN}$ and a screw diameter of $25 \mathrm{~mm}$ was used. Injection is conducted here in the conventional way, that is, via the plastification unit of the injection molding machine. Through the hot runner gating system, the melt flows into the cavity outside the mold axis. The hot runner at the same time prevents the melt from flowing back from the cavity during compression. Figure 3 shows a schematic illustration of the new mold technology.

Variotherm Tempering. To allow for variotherm tempering, which is required by the process, the experimental injection mold features electric heating elements connected to 

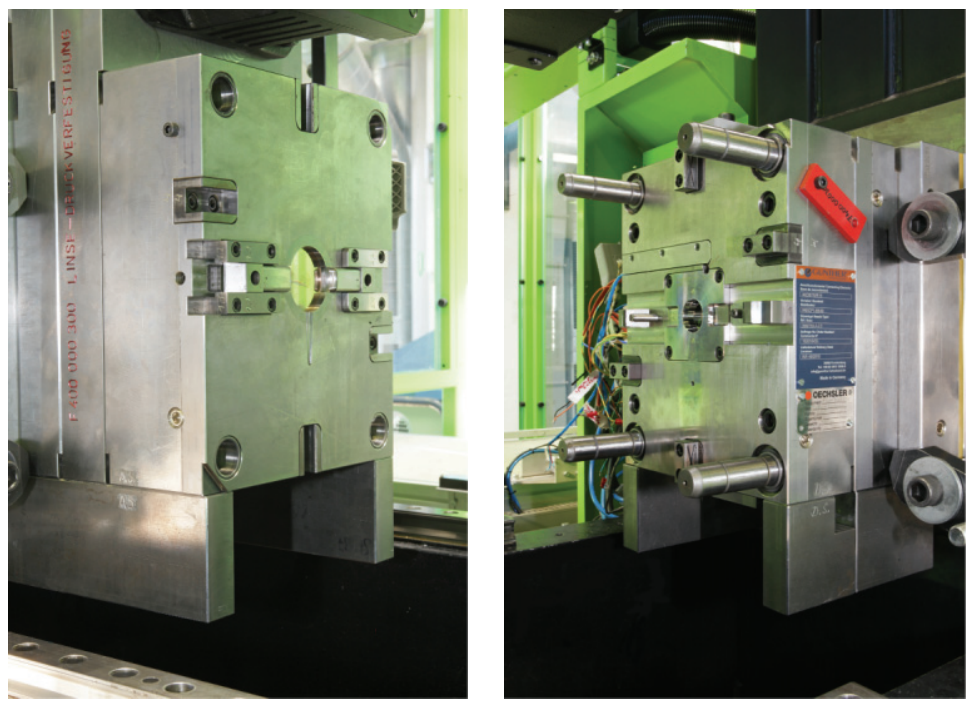

Figure 2: New mold technology for CIS integrated in the injection molding process left: ejection side with driven, temperature-controlled piston for compression application right: nozzle side with form-fitted heating and cooling, as well as sensor technology for temperature and pressure control.

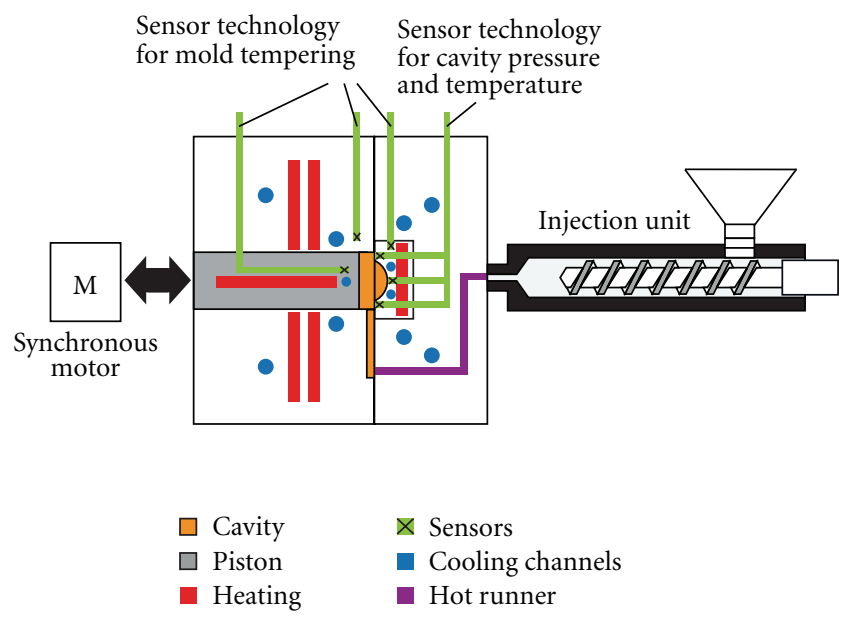

FIgURE 3: Schematic illustration of the new mold technology.

temperature sensors that are placed close to the cavity (see Figure 3). For fast heating, the nozzle side of the mold is equipped with a heating capacity of $700 \mathrm{~W}$, while the ejection side features a capacity of $8000 \mathrm{~W}$ because of its large dimensions to be heated. Furthermore, conventional tempering channels are positioned near the cavity to quickly cool down the mold to demolding temperature by means of constant water-media tempering. A short heating period is achieved by discontinuing the media tempering close to the cavity by means of a HB-Therm, Switzerland, tempering unit equipped with a pilot valve during the heating and compression phase.

Rapidly achieving the necessary, homogeneous experimental temperatures of up to $250^{\circ} \mathrm{C}$ within the cavity is one of the great challenges here. This calls for a simulation process to lay out the thermal conditions in the entire mold, mainly to estimate the homogeneity of temperature distribution and the heating performance required locally. The data obtained is to be verified too, in experiments to determine the temperature distribution in the cavity of the experimental injection mold.

Pressure Control. Densification moreover requires precise pressure control over the entire process. On the one hand, the defined pressures are to be applied and maintained precisely, while, on the other hand, isochoric process control (constant cavity volume by position control of the moving components) shall be possible in order to avoid excess compression, along with demolding under residual pressure. Pressure is therefore applied by a piston driven by a servo motor (see Figure 3). It is placed at the center of the mold and acts on the entire surface in the ejector half of the molded part. The temperature-related test pressures determined in the previous material characterizations in order to achieve or exceed the glass transition range can be achieved by this technique. Therefore, the process control is possible by regulation of cavity pressure. Here, the sensor is placed directly at the nozzle side in the cavity near the gate.

Additionally, pressure and temperature sensors that sit in the cavity provide for the control of the process by an external control unit and enable the determination of the molding characteristics. The piston from inside the cavity demolds the part.

3.3.2. Test Specimen. A circular disk serves as a specimen (Figure 4). To determine, by photoelasticimetry, the residual stresses that exist within the component, it its necessary for the specimen to have parallel faces. The molding moreover has thick walls, which, due to the high cooling gradient over the wall thickness, will probably feature unfavorable 

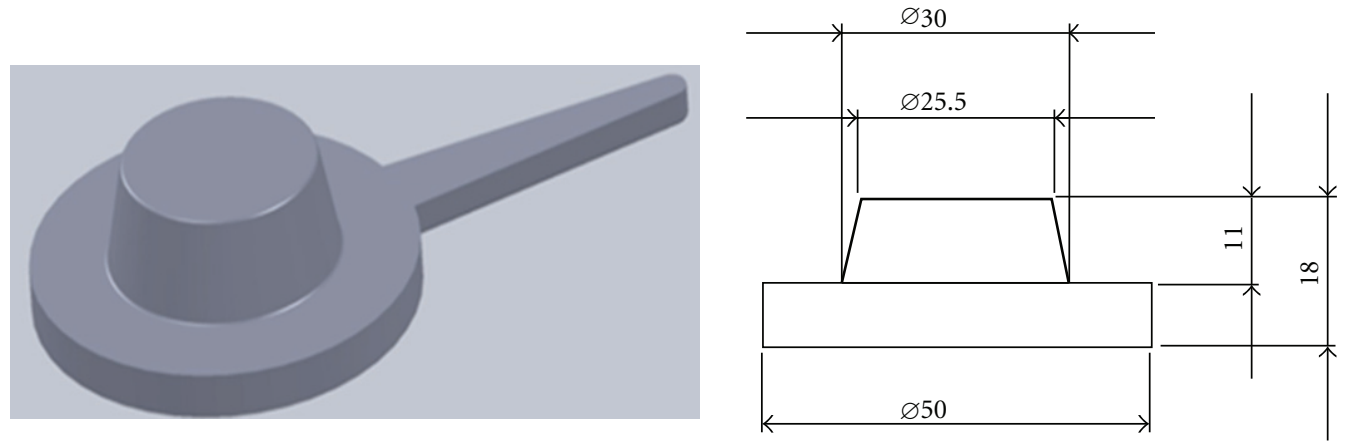

Figure 4: Circular disk specimen (left) and cavity dimensions at room temperature (right).

component characteristics, that is, considerable residual stress, if produced by conventional injection molding. On the other hand, this type of specimen is well suited to clearly show the benefits included in the CIS technique.

3.3.3. Experimental Procedure. Before the specimen is produced, the material is predried under vacuum at $80^{\circ} \mathrm{C}$ for $24 \mathrm{~h}$ to reduce the residual moisture below $0.05 \%$ per weight which was evaluated by the Karl-Fischer titration. It is then plasticized in an injection molding machine by the conventional way. In addition to moderate plastification settings, a melt temperature of $270^{\circ} \mathrm{C}$ is chosen. Not only does this setting have to safeguard error-free filling of the cavity but it must moreover provide for short adapting time, until the entire molding has reached the lower test temperature of $170^{\circ} \mathrm{C}$. The investigations start from the adapting time of $420 \mathrm{~s}$ calculated by the Abaqus simulation program, required to reach the molding temperature of $170+6{ }^{\circ} \mathrm{C}$. To counteract the thermal shrinkage over the adapting period, the plastification unit applies moderate packing pressure.

Prior to filling, the electric heating elements heat the mold to $170^{\circ} \mathrm{C}$ test temperature and keep the temperature constant. Following the period of adapting, compression pressure is applied and maintained for $3 \mathrm{~s}$. Pressures range from below the beginning until the end of glass transition, according to the findings obtained in material characterization. The specimen is finally submitted to cooling in an isochoric process, that is, with the volume kept constant. A schematic diagram of the process of CIS is shown in Figure 5.

Further investigations focused on the influence of the adapting time on part properties. For this purpose, three adapting times were applied, that is, the adapting time calculated by the Abaqus simulation program, one below, and one above this (Table 3 ).

For the purpose of comparing the part properties of the CIS specimens to the characteristics of parts produced in a standard injection molding process, a reference specimen was generated by conventional injection molding. In this process, melt temperature was $300^{\circ} \mathrm{C}$, mold temperature was at a low $100^{\circ} \mathrm{C}$, packing pressure amounted to 2400 bars for $40 \mathrm{~s}$, and cooling took $120 \mathrm{~s}$.

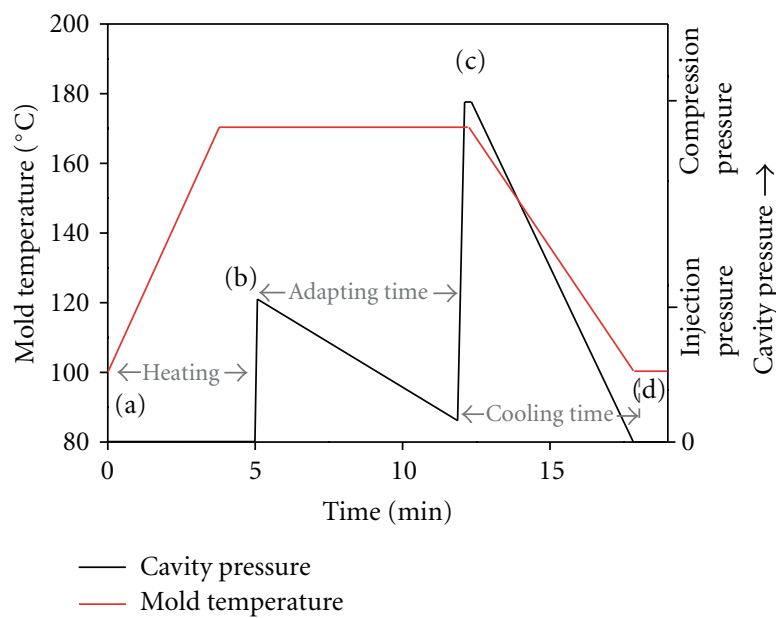

FIGURE 5: Schematic diagram of the process of CIS: (a) mold heating starts; (b) melt injection and begin of adapting time; (c) compression and shortly afterwards start of mold cooling (isochoric process); (d) demolding.

TABLE 2: Experimental settings for CIS with varying compression pressure.

\begin{tabular}{lc}
\hline Melt temperature $\left[{ }^{\circ} \mathrm{C}\right]$ & 270 \\
Test temperature (high mold & 170 \\
temperature) $\left[{ }^{\circ} \mathrm{C}\right]$ & \\
Adapting time $[\mathrm{s}]$ & 420 \\
Compression pressure [bars] & $600-1000$ in steps of 100 \\
Packing time of compression $[\mathrm{s}]$ & 3 \\
Process during cooling $[-]$ & Isochoric \\
Cooling time $[\mathrm{s}]$ & 360 \\
$\begin{array}{l}\text { Demolding temperature (low mold } \\
\text { temperature) }\left[{ }^{\circ} \mathrm{C}\right]\end{array}$ & 100 \\
\hline
\end{tabular}

\subsection{Methods of Analysis}

3.4.1. Determination of the Temperature Distribution of the Mold. To achieve the necessary homogeneous experimental temperature of $170^{\circ} \mathrm{C}$ within the cavity is one of the great challenges. For this purpose, the temperature distribution during the heating phase was examined by means of an 
TABLE 3: Experimental settings for CIS with varying adapting time.

\begin{tabular}{|c|c|c|}
\hline Melt temperature $\left[{ }^{\circ} \mathrm{C}\right]$ & & 270 \\
\hline $\begin{array}{l}\text { Test temperature (high mold } \\
\text { temperature) }\left[{ }^{\circ} \mathrm{C}\right]\end{array}$ & & 170 \\
\hline Adapting time $[\mathrm{s}]$ & 180 & 420 \\
\hline Compression pressure [bars] & & 800 \\
\hline Process during cooling $[-]$ & & sochoric \\
\hline Cooling time $[\mathrm{s}]$ & & 360 \\
\hline $\begin{array}{l}\text { Demolding temperature (low mold } \\
\text { temperature) }\left[{ }^{\circ} \mathrm{C}\right]\end{array}$ & & 100 \\
\hline
\end{tabular}

infrared thermographic camera, PIR uc 180, by InfraTec $\mathrm{GmbH}$ with a thermal resolution of $0.08 \mathrm{~K}$ at $30^{\circ} \mathrm{C}$. The measurements were analyzed with the thermal imaging software IBRIS 3, by Infra-Tec GmbH. Because of the low emission coefficient of metals, that is, 0.2 to 0.5 , its variation with temperature [10], and the reflections of the thermal radiation due to the reflective surfaces of the mold, the relevant parts of the mold were masked with polyamide tape.

3.4.2. Photoelastic Investigations. Photoelasticimetry is aimed at visualizing a transparent material's birefringence. In amorphous materials, this is caused either by applying stress from the outside, by residual stresses or orientations due to the production process. A transparent birefringent sample placed in the light path of the lamp, between polarizer and analyzer, generates an image showing changes in characteristic colors-according to the Michel-Lévy color chart, in the case of white light-which represents the existing stresses and orientations. Lines of the same color are called isochrones. The distribution and number of isochrones gives an indication as to the quality of the stresses that exist within a component. Additionally, quarter-wave plates may be used, which circularly polarize the light and filter disturbing isoclines [11].

All samples were examined in a photoelastic testing device for large surfaces supplied by Dr. Schneider Messtechnik at circularly polarized white and monochromatic lights, and pictures were taken with a digital camera (Canon EOS 5D Mark II). Based on the number of isochrones at the circular disk's central area, which is approximately $18 \mathrm{~mm}$ thick, the pictures were then evaluated. Over the thickness, that is, height, of the component and via the materialspecific photoelastic constant, the isochrone order can be determined by using the Michel-Lévy color chart and the principal stress difference can be calculated. An external micrometer screw gauge served to measure the sample height. For polycarbonate, the photoelastic constant ranges between 7.5 and $10 \mathrm{~N} / \mathrm{mm}^{*}$ order. For this purpose, the number of repetitions of the colors that are characteristic for order transition are counted and-by employing the main equation of photoelasticimetry

$$
\sigma_{1}-\sigma_{2}=\frac{S}{d} \cdot n
$$

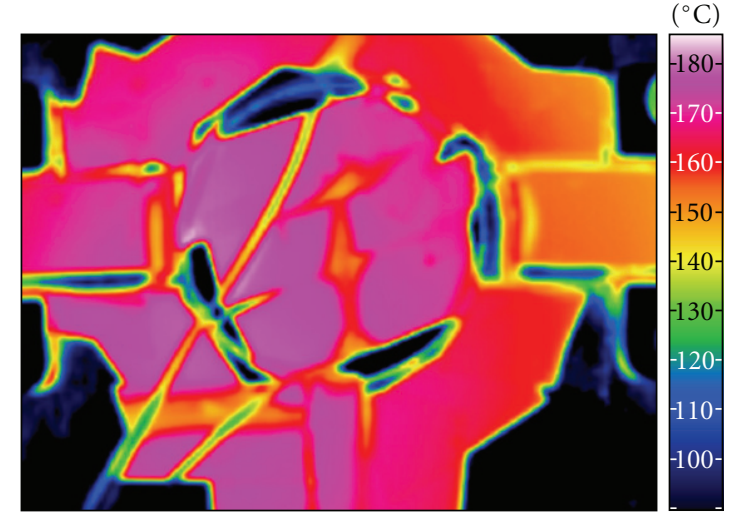

Figure 6: Temperature distribution at the ejection side at $170^{\circ} \mathrm{C}$ test temperature, analyzable in terms of quality and determined by a thermographic camera in experimental investigations.

with $S=$ photoelastic constant, $d=$ component height, $n=$ isochrone order-transformed into the maximum principal stress difference [1]. For the employed polycarbonate, Makrolon LQ 2647, a photoelastic constant $S$ was determined of $8.2 \mathrm{~N} / \mathrm{mm}^{*}$ order. Because packing times and thus relaxation times at melt temperature are very long, orientationinduced birefringence can be assumed to have no significant effect on densification [2].

3.4.3. Determination of Dimensional Accuracy. In order to examine the effects of compression pressure and process control on the geometric properties of the specimen, the dimensional accuracy was determined after the parts had been demolded and cooled down to room temperature. For this purpose, the height of the truncated cone on the specimen central area was measured three times with an external micrometer screw gauge. The measuring range was $25 \mathrm{~mm}$ while resolution was $0.01 \mathrm{~mm}$, and the difference between the average value and the mold size was determined. For a measuring range up to $25 \mathrm{~mm}$, the margin of error in external micrometer screw gauges is $4 \mu \mathrm{m}$ according to the German Industrial Standard (DIN 863-1) [12].

\section{Results and Discussion}

4.1. Determination of the Temperature Distribution of the Mold. Figure 6 shows the qualitative temperature distribution at the ejection side of the experimental injection mold, determined by means of a thermographic camera at $170^{\circ} \mathrm{C}$ test temperature. As can be seen, the distribution of temperature in the area of the piston is largely homogeneous. Only a slight temperature gradient appears over the horizontal expansion of the mold. This undesirable effect is a consequence of the marginal differing positions of the temperature sensors, which regulate the electric heating elements positioned on each side of the piston.

4.2. Determination of the Adiabatic pvT Behavior. Figure 7 shows an adiabatic $p v T$ diagram representing the material 


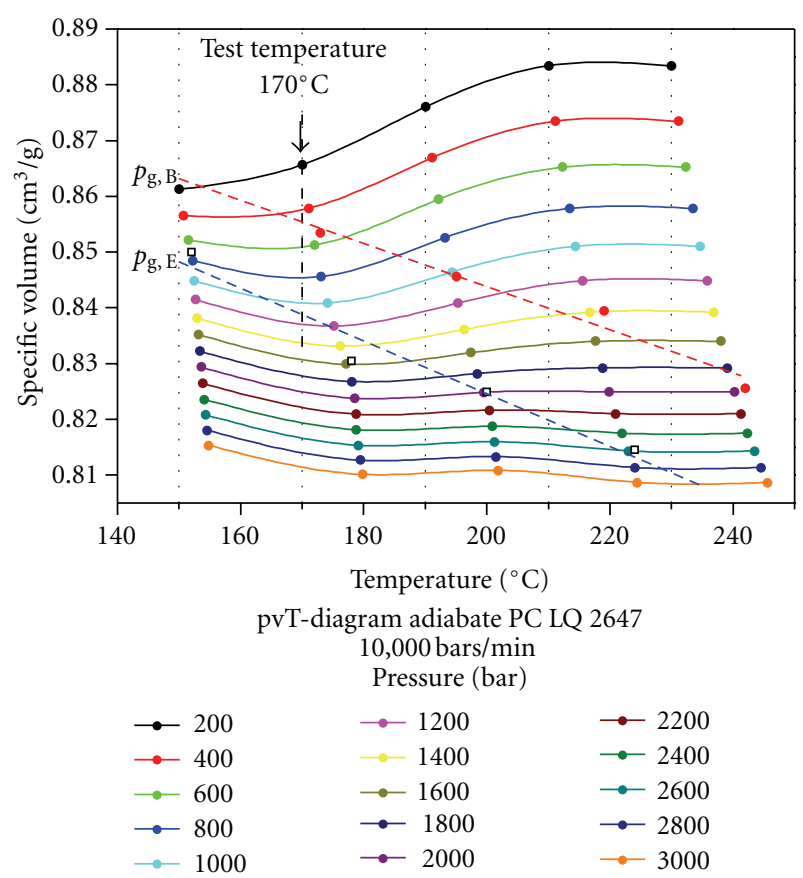

Figure 7: Adiabatic $p v T$ behavior of PC Makrolon LQ $2647 p_{g, B}$ : beginning of glass transition (dashed red line) $p_{g, E}$ : end of glass transition (dashed blue line).

characterization for the PC Makrolon LQ 2647 that is subject to the investigations. Unlike the case in a standard $p v T$ diagram, isobars do not describe the course of isobaric cooling here, but they rather connect the specific volumes achieved at the same pressure. This diagram is thus dependent on the process path, that is, at which temperature compression of the melt takes place. If compared to isobaric measurement, higher specific volumes are achieved. Only under high melt temperatures, the higher chain mobility incurred leads to the same values as in isobaric $p v T$ measurements [13]. Moreover, this adiabatic $p v T$ diagram also includes the limits of the range of glass transition (beginning of glass transition $p_{g, B}$, end of glass transition $p_{g, E}$ ) representing the basis for the process pressures defined in the design of experiments. The limits were determined by calculating the compression moduli as described by Breuer and Rehage [9]. At the experimental temperature chosen of $170^{\circ} \mathrm{C}, p_{g, B}$ is at just under 500 bars and $p_{g, E}$ is at approx. 1100 bar. 600 bar of compression pressure thus corresponds to just above $p_{g, B}$ of experimental pressure. As compression pressure rises up to $1000 \mathrm{bar}$, the range of glass transition is passed until close to $p_{g, E}$ (Table 2).

\subsection{Influence of Compression Pressure on Part Properties}

4.3.1. Residual Stresses. Figure 8 presents photographs of the photoelastic behaviors of specimens produced by CIS at varying compression pressures. With increasing compression pressure until 900 bar, the number of isochrones declines. A compression pressure of $1000 \mathrm{bar}$, which corresponds to the end of the glass transition $p_{g, E}$ at a temperature of $170^{\circ} \mathrm{C}$, leads to a comparable pattern as reached with 900 bar.

The maximum principal stress differences of specimens produced by CIS in dependence of compression pressure, evaluated from the photoelastic images, are demonstrated in Figure 9. Generally speaking, all specimens produced by CIS show lower numbers of isochrones, which means lower maximum principal stress differences compared to the injection molded reference specimen, which is represented by the dashed line. If compression pressure of 600 bars is just a little bit higher than $p_{g, B}$, principal stress differences are slightly under those of the reference specimen, which is nearly $5 \mathrm{~N} / \mathrm{mm}^{2}$. With increasing compression pressures in the glass transition range, the principal stress differences decrease significantly to about the half with $2.3 \mathrm{~N} / \mathrm{mm}^{2}$ at 900 bar. Towards the end of glass transition $p_{g, E}$, the number of isochrones remains approximately the same, and consequently the maximum principal stress difference too.

4.3.2. Dimensional Accuracy. Investigations conducted on dimensional accuracy show that the level of deviation from the mold dimensions is significantly lower in the CIS specimens than that in the injection molded reference specimen (Figure 10). As the compression pressure increases in the glass transition range, the dimensions of the sample approach the dimensions of the mold as a result of increased molecular densification, reaching $0.01 \mathrm{~mm}$ at 1000 -bar compression pressure. However, in order to reduce residual stress, demolding under residual pressure must be prevented. Consequently, further investigations are due to comparing the dimensions of molded parts at demolding temperature.

\subsection{Influence of Temperature Adapting Time on Part Properties}

4.4.1. Residual Stresses. Figure 11 shows the photoelastic behavior in dependence on adapting time. It can be clearly seen that the number of isochrones decreases with longer adapting times. In Figure 12, the maximum principal stress difference in dependence on adapting time is illustrated. The principal stress difference is strongly dependent on the adapting time and therefore on the temperature gradient in the melt at the time of compression. If the adapting time is too short to reach a homogeneous temperature field within the molten part, the solidification is displaced spatially and temporally. The surface areas solidify first while the part's core remains liquid. The core solidifies later with higher pressures or during the cooling process. Therefore, short adapting times lead to high numbers of isochrones and maximum principal stress differences. With increasing adapting time, both the numbers of isochrones and the maximum principal stress difference decrease.

4.4.2. Dimensional Accuracy. The effect of the adapting time on the dimensional accuracy is shown in Figure 13. Just as the maximum principal stress difference, the dimensional accuracy decreases with longer adapting times. This means 


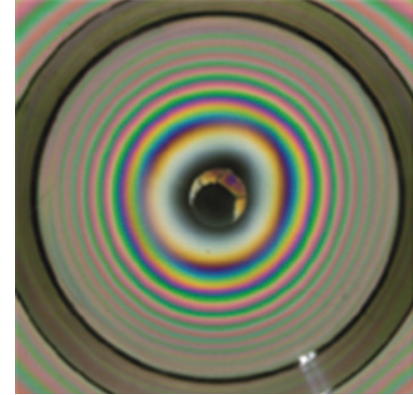

Compression pressure: 600 bar

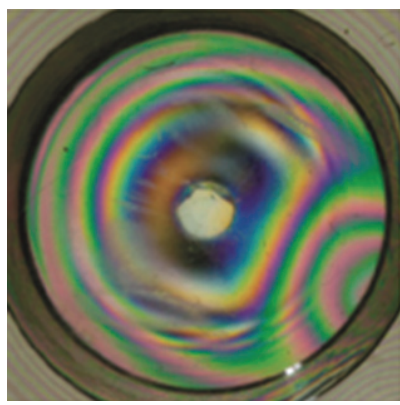

Compression pressure: 900 bar

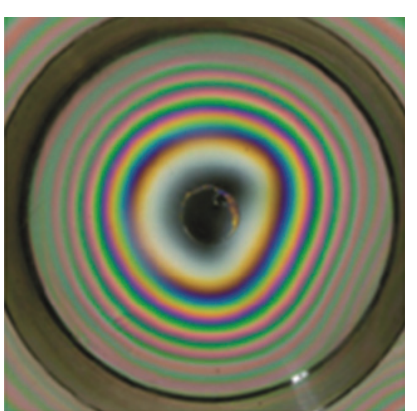

Compression pressure: 700 bar

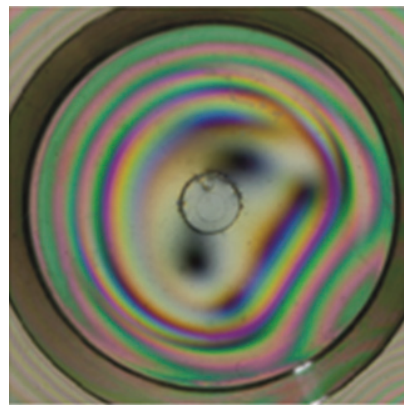

Compression pressure: 1000 bar

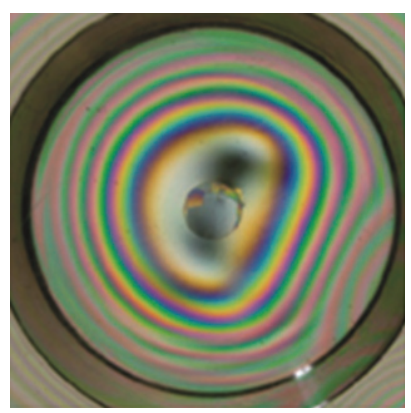

Compression pressure: 800 bar

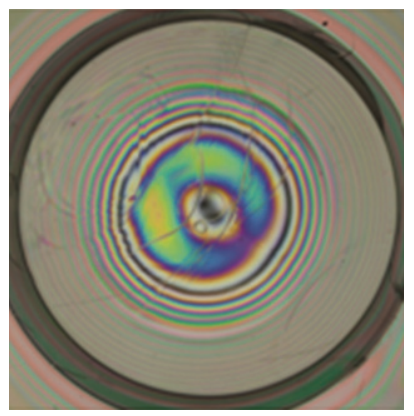

Injection molded part $\underline{5 \mathrm{~mm}}$

Figure 8: Photoelastic behavior in dependence on compression pressure.

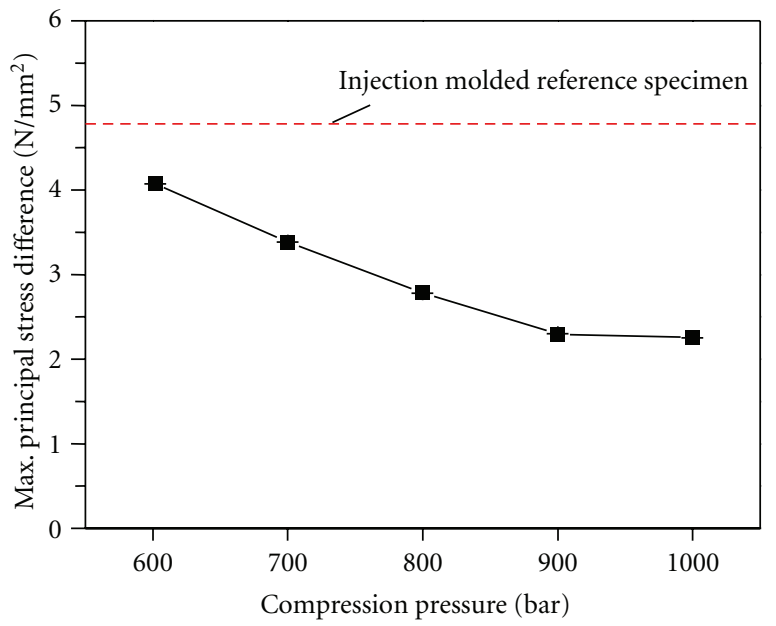

Figure 9: Maximum principal stress differences in dependence on compression pressure.

that homogeneous temperatures within the part during compression are important for appropriate part dimensions.

4.5. Optimization of Cycle Time. The cycle times of the conducted trials are mainly composed of mold heating time (5 $\mathrm{min})$, adapting time $(7 \mathrm{~min})$, and cooling time $(6 \mathrm{~min})$. The rest of the cycle time is made up of comparably short times, such as demolding, applying pressure, and moving the mold. Altogether, they take less than one minute. For the complete process, relatively long cycle times of nearly $20 \mathrm{~min}$ are required. In contrast, longer adapting times lead to better part properties but extend the cycle time. In order to establish

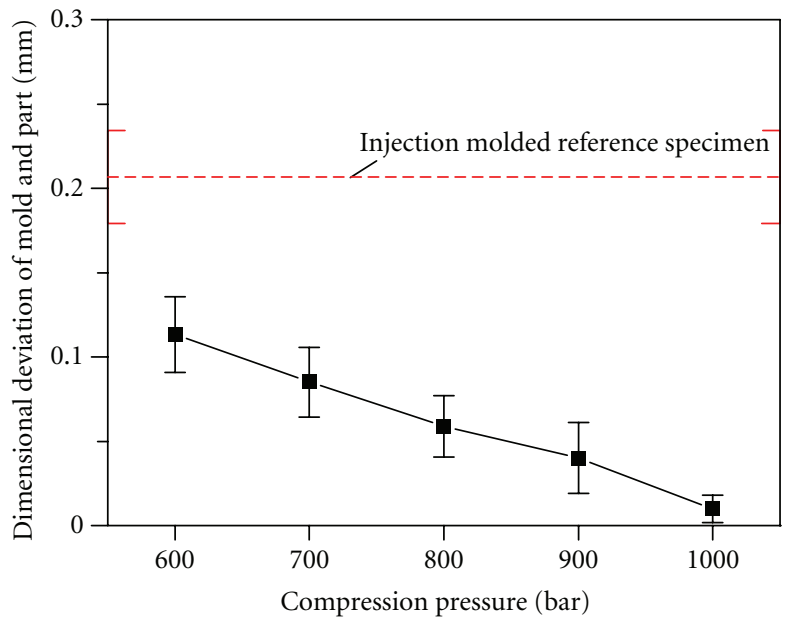

FIgure 10: Dimensional deviation between mold and part in dependence on compression pressure.

CIS as a competitive production process, it is necessary to reduce cycle time. There may be several options of achieving this aim.

The first is to reduce the temperature difference between the injected melt and the heated mold. For this, melt temperature could be reduced or mold temperature could be increased. If stepping up the mold temperature, higher solidification pressures during the process will be necessary. This will lead to higher mechanical stresses in the mold, and process control will become more difficult to handle. On the other hand, reducing the melt temperature will lead to higher melt viscosity, which will increase the difficulties in the injection process. 


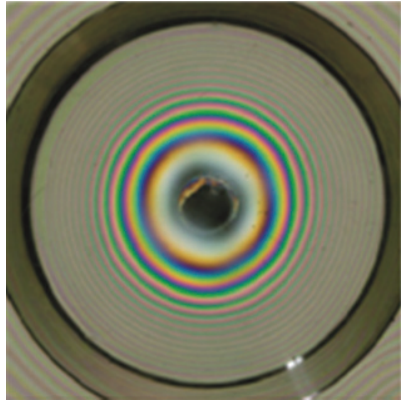

Adapting time: $180 \mathrm{~s}$

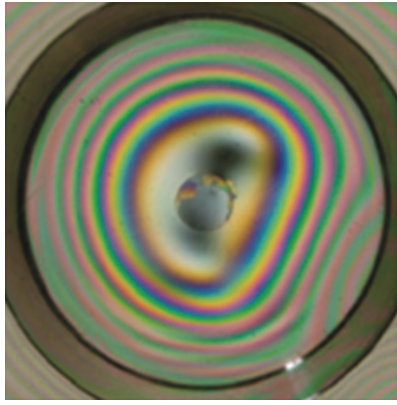

Adapting time: $420 \mathrm{~s}$

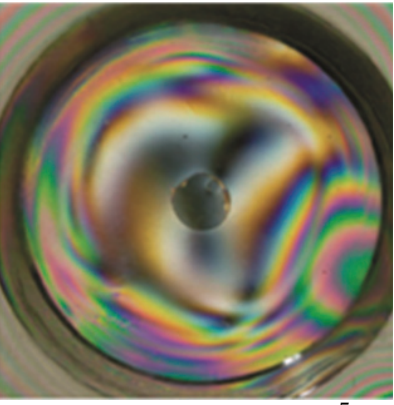

Adapting time: $780 \mathrm{~s} \quad \underline{5 \mathrm{~mm}}$

FIgURE 11: Photoelastic behavior in dependence on adapting time (800-bar compression pressure).

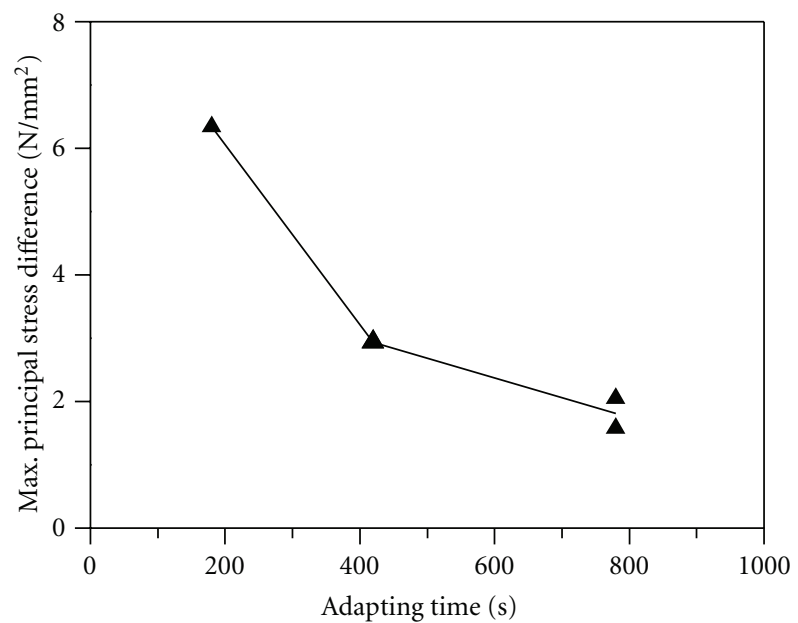

FIGURE 12: Maximum principal stress differences in dependence on adapting time (800-bar compression pressure).

The second option is to improve the heating and cooling rate of the mold during the variotherm process. Therefore, more heating power or other technologies such as variotherm fluid temperature control with two circuits could be useful.

Further progress in reducing the cycle time could be made by changing the process. It should be possible to inject the melt before or during the mold heating process. First simulations of this approach show that it should be possible to reach trial temperature of the melt in the part's core at the same time when mold heating is completed. This means that extra adapting time would be no longer necessary. However, specific difficulties at this approach such as the solidification and remelting of the melt in the exposed or thin-walled regions have not been considered yet. Further investigations relating to this point are planned.

\section{Conclusions}

The innovative process variant of compression-induced solidification (CIS), integrated into the injection molding process, is a novel way of production for thermoplastic components. The technique connects the benefits of the

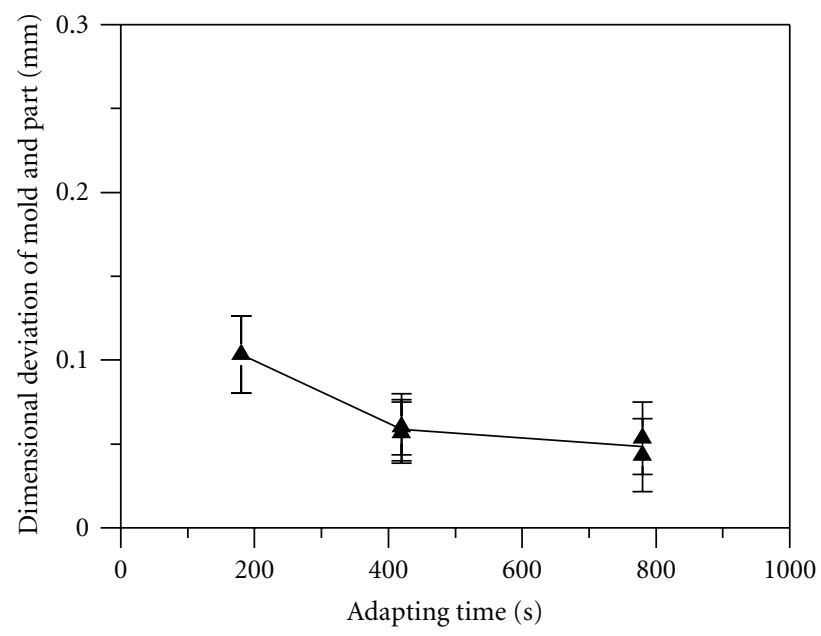

FIGURE 13: Dimensional deviation between mold and part in dependence on adapting time.

injection molding manufacturing process in terms of cost efficiency and freedom of design to excellent component properties, particularly for thick-walled optical components. Certainly, the costs of the CIS cannot be unattended which are somewhat higher due to the necessary additional equipment, for example, heating and compression resources, and the more complex mold in comparison to the conventional injection molding process. The study at hand shows that the CIS technique is suitable to generate components with significantly reduced residual stresses and with high dimensional stability, while featuring an optimum level of compression pressure at the end of glass transition range. Moreover, great benefits can be reached by extension of the adapting time to reach homogenous temperatures. However, the extension of cycle time involved has to be considered. By variation of temperatures and process control, future investigations are due to find the causes of the remaining residual stress and thus to further optimize component properties.

\section{Acknowledgments}

The authors gratefully acknowledge the German Research Foundation (DFG) for the financial support and the 
industrial partners Engel Austria GmbH, Austria, HB Therm AG, Switzerland, and Bayer Material Science AG, Germany, for providing the processing equipment and material.

\section{References}

[1] N. Rudolph, Druckverfestigung amorpher Thermoplaste [Ph.D. thesis], Institute of Polymer Technology, Friedrich-AlexanderUniversität Erlangen-Nürnberg, 2009.

[2] S. Bölinger, Spritzgießen und spritzprägen von kunststoffoptiken [Ph.D. thesis], Institute of Plastics Processing, RWTH, Aachen, Germany, 2001.

[3] P. Thienel, Der formfüllvorgang beim spritzgießen von thermoplasten [Ph.D. thesis], Faculty of Mechanical Engineering, RWTH, Aachen, Germany, 1977.

[4] N. Rudolph, G. W. Ehrenstein, and T. A. Osswald, "New insights in pvT-behavior for high precision in injection molding," in Proceedings of the 68th Annual Technical Conference of the Society of Plastics Engineers (ANTEC '10), pp. 890-895, Orlando, Fla, USA, May 2010.

[5] R. Y. Chang, Y. C. Hsieh, and C. H. Hsu, "On the pvT and thermal shrinkage for the injection molding of a plastic lens," in Proceedings of the 56th Annual Technical Conference (ANTEC '98), pp. 574-578, Atlanta, Ga, USA, April 1998.

[6] N. Rudolph, K. Vetter, and I. Kühnert, "Temperature change by compression and expansion of polymer melts," Journal of Plastics Technology, vol. 6, no. 4, pp. 250-277, 2009.

[7] N.N.: ISO Datasheet, Makrolon LQ2647, Bayer MaterialScience AG, Edition 2010.

[8] N. Rudolph, "Solidification by pressure instead of cooling," Kunststoffe International, vol. 7, pp. 58-61, 2011.

[9] H. Breuer and G. Rehage, "Zur thermodynamik der glasigen erstarrung," Kolloid-Zeitschrift \& Zeitschrift für Polymere, vol. 216-217, no. 1, pp. 159-179, 1967.

[10] J. Merchant, Infrarot-Temperaturmessung, Newport Electronics $\mathrm{GmbH}$.

[11] H. Wolf, Spannungsoptik, Springer, Berlin, Germany, 1976.

[12] DIN 863-1: DIN 863 Teil 1 April 1999. Prüfen geometrischer Größen-meßschrauben-teil 1: bügelmeßschrauben, Normalausführung, Begriffe, Anforderungen, Prüfung.

[13] N. Rudolph, I. Kühnert, and G. W. Ehrenstein, "Compression induced solidification (CIS) - a novel injection molding strategy for high precision parts," in Proceedings of the 67th Annual Technical Conference of the Society of Plastics Engineers (ANTEC '09), pp. 2087-2091, Goa, India, June 2009. 

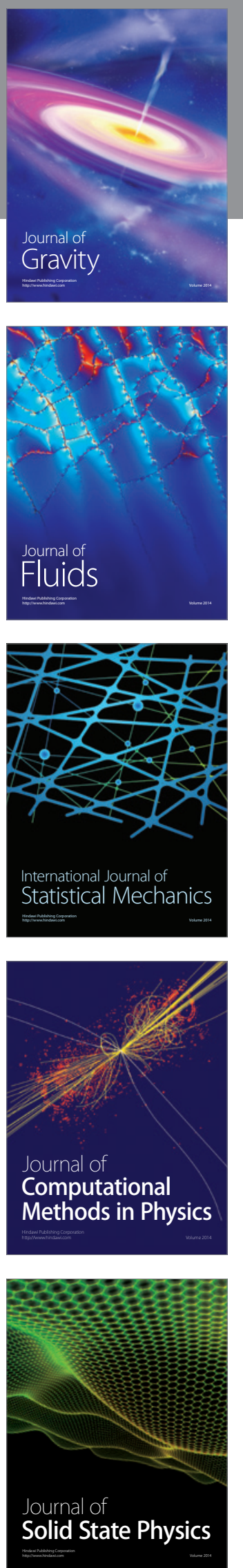
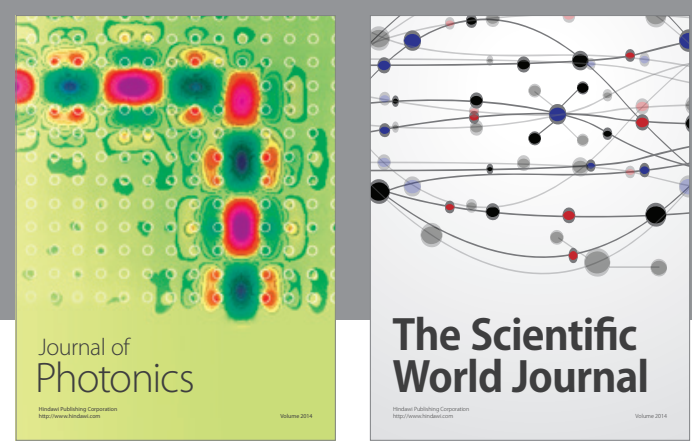

The Scientific World Journal

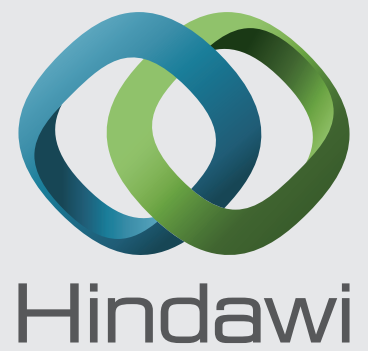

Submit your manuscripts at http://www.hindawi.com
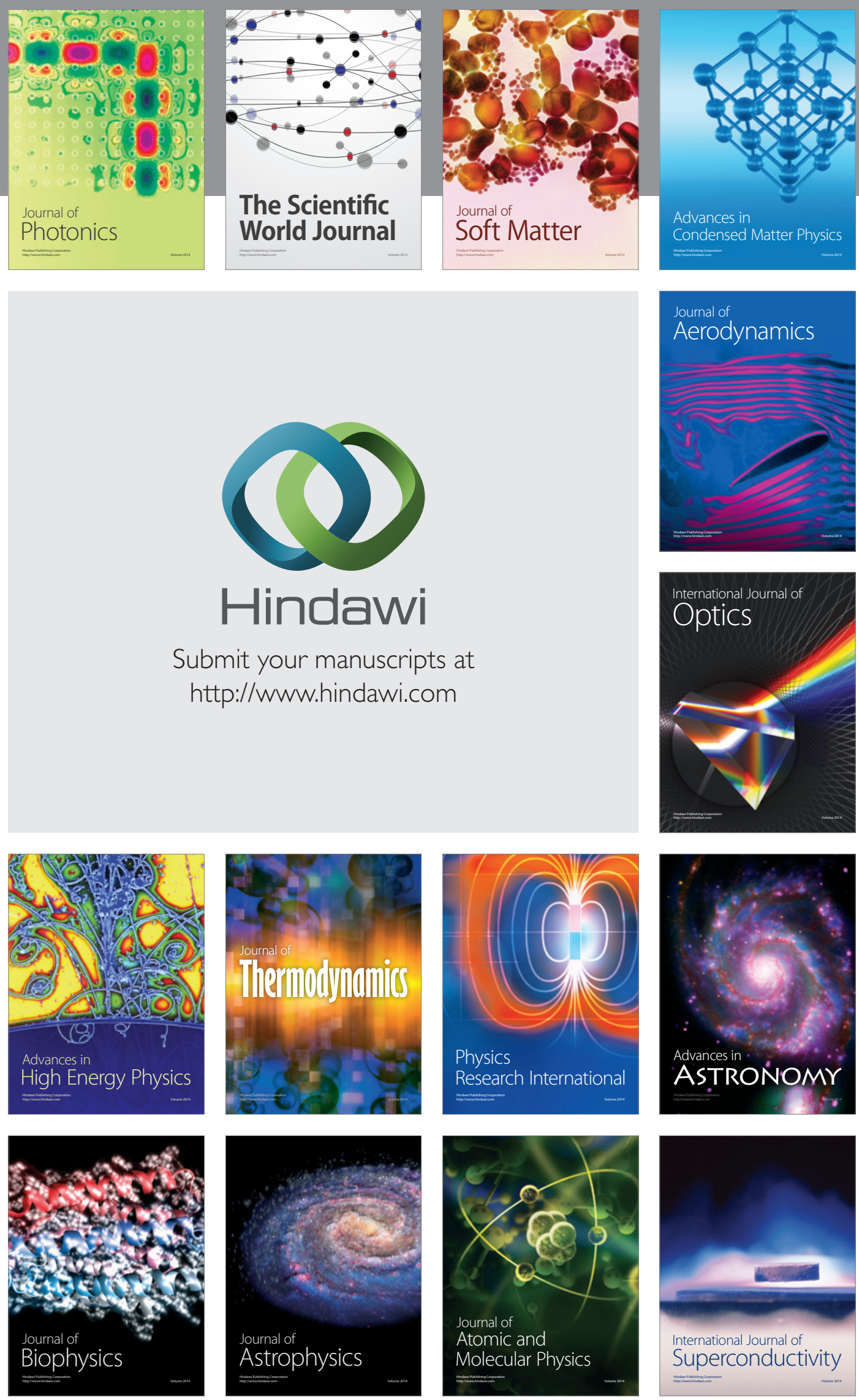
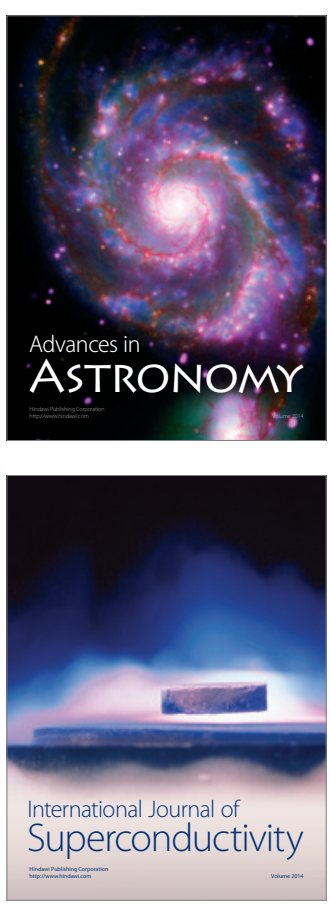